\title{
Supervised regression modelling for mitigation of four-wave mixing in dense wavelength-division multiplexing systems
}

\author{
${ }^{1}$ Venkatesan K., ${ }^{2}$ Chandrasekar A. and ${ }^{3}$ Ramesh P. G. V. \\ ${ }^{1}$ Department of Electronics and Communication Engineering, St. Joseph's College \\ of Engineering, Chennai, India venkatsri21@gmail.com \\ ${ }^{2}$ Department of computer science and Engineering, St. Joseph's College of \\ Engineering, Chennai, India \\ ${ }^{3}$ Department of Electronics and Communication Engineering, St. Joseph's Institute of \\ Technology, Chennai, India
}

Received: 06.10 .2020

\begin{abstract}
A recent global crisis associated with COVID-19 has encouraged millions of people to work from home, thus causing a drastic increase in overall network traffic, data-rate requirements and end network capabilities. This has also produced more noise, cross-talk and undesirable optical-fibre nonlinearities, especially a fourwave mixing (FWM) effect that deteriorates performance of dense wavelengthdivision multiplexing (DWDM) systems. A presence of FWM in the DWDM systems imposes increasing complexity and latency of networks, and decreases their spectral efficiency. In its turn, this degrades efficient utilization of optical bandwidth. To mitigate the above problems, we suggest a supervised regression modelling (SRM). A relevant SRM-DWDM approach performs self-parametric optimization of the DWDM systems with machine-learning techniques and finds real trade-offs among various factors that affect the FWM. Our model reduces complexity of modelling and computational time, resulting in accurate and reliable prediction of parameter values. We also evaluate the performance of our SRMDWDM technique by comparing its data with the iterative results obtained for different parameters (e.g., output signal-to-noise ratio, Q-factor, signal power and noise power). Finally, we specify the procedures necessary for global optimization of DWDM systems.
\end{abstract}

Keywords: supervised regression algorithm, machine learning, DWDM, four-wave mixing, Q-factor, optical signal-to-noise ratio.

UDC: 681.7

\section{Introduction}

Fibre-optic systems with dense wavelength-division multiplexing (DWDM) provide high transmission capacity, wide bandwidth and efficient use of bandwidth [1]. Now it is important to ensure high reliability of DWDM systems to achieve high-quality data transmission in longdistance communication networks [2]. Increasing number of channels in a DWDM system makes its structure more complex and produces a number of linear and nonlinear issues [3]. One can mention in this relation nonlinear light scattering, Kerr nonlinearities and parabolic nonlinearities, along with amplified spontaneous emission. Moreover, there are such effects as stimulated Raman scattering [4], four-wave mixing (FWM) and cross-phase modulation [5], which also affect data transmission and optical signal-to-noise ratio (OSNR) in long-haul fibre-optic systems [6].

A presence of FWM in an optical DWDM system can affect notably the quality of signal during its transmission. Various parameters such as OSNR, bit-error rate (BER) and Q-factor are helpful to determine the effect of FWM and its influence on the system. Mitigation of the FWM in 
DWDM systems [7] has been discussed in relation to various system functions such as number of channels [8], bit rate [9], channel spacing [8], modulation format [9] and duty cycle [10]. The influence of FWM in many waveguide devices is observed while varying pump power and detuning light wavelength [12]. A reduction of FWM achieved using different modulation formats in long-haul DWDM transmission systems has also been discussed and the system performance has been evaluated basing on the OSNR, the Q-factor and the BER [13]. A turn-to-zero (RZ) modulation format has proven to be less affected by the nonlinearities. It can provide a better Qfactor, lower distortion and lower noise [14]. Analysis of optical amplifiers for the DWDM systems under conditions of different channel spacings, input powers, optical gains and opticalfibre core sizes has provided estimation of such system factors as BER, Q-factor, OSNR and output signal power. This also yields in quantitative measures for the nonlinearities and cross-talk and predetermines the final values of guaranteed quality of transmission $[16,17]$.

Traditional methods employed for calculating optical networks require much time and increase system complexity, which hinders their use while achieving global optimization [18, 19]. These limitations can, in principle, be overcome with novel approaches, e.g. the techniques based upon artificial intelligence and machine learning (ML). The latter can provide superior solutions in relation to the complexity issues [20]. Detection, identification and management of failures in optical networks have already been studied using the ML techniques [21]. An ML-based reduction of OSNR, input power and noise figures and estimation of the quality of transmission in the DWDM networks have been discussed in Ref. [22]. The ML procedures and their efficiency for various communication fields such as software-defined networks [23], Internet of Things [24], wireless sensor networks [25], cognitive radios [26], wireless networks [27], computer networks [28] and traffic classification [29] have been studied.

One can outline the following problems to be solved in this direction: (i) design of long-haul optical DWDM systems with wider bandwidth and larger number of channels, which involves complex-network management and difficulties in collecting data from enormous number of monitoring points, and (ii) parametric optimization for mitigating fibre nonlinearities, especially the FWM effect. Sometimes these problems represent real challenges and cannot be easily solved through simple iterative simulations under diverse practical constraints. Therefore, a real-time environment requires efficient ML-based prediction approaches for self-optimization of highcapacity DWDM systems, following from user requirements, efficient network management and avoiding of bandwidth crunch.

In this work, we suggest a self-parametric optimization via an ML-based supervised regression model (SRM) for a high-capacity DWDM system in the presence of FWM. A so-called SRM-DWDM technique is designed and verified. It achieves higher data rate and provides better understanding of the parameters that influence the FWM. The suggested SRM-DWDM method is analyzed for various FWM-influencing parameters such as input power, channel spacing, modulation format, data rate and gain of optical amplifier. The final aim is user requirements of larger bandwidth and efficient utilization of channel capacity. These tasks can be performed under conditions of reduced system complexity and acceptable iterative runtime.

The article is organized as follows. Section 2 discusses in detail the related work on the subject. Section 3 provides the fundamentals of FWM and its influence on the DWDM systems. In Section 4 we describe a conventional (iterative) simulation setup for DWDM systems. Detailed information on our SRM-DWDM approach and the processes involved in its operation is gathered in Section 5. In Section 6 we report on the tests of our SRM-DWDM system and parametric 
observations of various system functions and factors mitigating the FWM effect. Finally, Section 7 concludes and discusses a future scope.

\section{Related work}

There are several nonlinear impairment factors that deteriorate the efficiency of DWDM due to their uncertainty. To estimate these uncertainties, the quality of transmission and design considerations must be considered. The design considerations derived from either a simple analytical model or an advanced DWDM-designing techniques can be used. Channel spacing, modulation format, input power, data rate and some other influencing parameters are required to estimate the quality of transmission through a DWDM system. The introductory studies have shown that such parameters such as the OSNR, the noise power, the signal power and the Q-factor reveal exponential dependences on the channel spacing, the input power and the modulation format at high data rates. For example, Ref. [22] mentions fluctuations in the quality of transmission that affect the spectral characteristics and thereby introduce deviations in the optimal values of FWM-mitigating factors.

The authors [7] have analyzed a case of low channel spacing and small number of input channels, when the utilization of bandwidth and the channel capacity are limited. In Ref. [8] it has been shown that a larger number of channels (a case of 80 channels) and ultra-narrow channel spacing $(0.25 \mathrm{~nm})$ make the design much more complex. The work [6] has discussed a DWDM system with chromatic dispersion, which achieves 1.28 Tbps data transmission with some limited factors such as channel spacing and transmission distance. Unlike this work, a $0.2 \mathrm{~nm}$ channel spacing with high inter-channel nonlinearities has been analyzed in Ref. [17]. It can achieve the gain ripple $1.70 \mathrm{~dB}$ and the noise figure $4 \mathrm{~dB}$. The author [3] has suggested a radio-over-fibre system, where optimization is achieved using an 8-channel WDM transmitter, a $75 \mathrm{GHz}$ channel spacing, a $0 \mathrm{dBm}$ input power and a $4 \mathrm{dBm}$ FWM power. Different modulation formats with variable duty cycle for various channel spacings in an EDFA structure have been discussed in Ref. [10].

The work [1] has studied different FWM-influencing parameters and a real trade-off between reduced FWM and low bandwidth accompanied with low channel capacity. The author [12] has constructed a DWDM system with high conversion efficiency but difficulties in attaining phasematching condition. A DWDM model has been proposed in Ref. [2] with a coherent modulation format and 3R system, which consumes about $90 \%$ transmission power at the receiver end. The author [16] has achieved the quality factor $30 \mathrm{~dB}$, the gain $29.4 \mathrm{~dB}$ and the eye closure $0.9 \mathrm{~dB}$ in the presence of serious inter-symbol interference. In the work [4] an efficient wavelength-division multiplexing system has been suggested for the case of symbol rate 50 Gbaud, quadrature phaseshift keying and a 16-QAM modulation format. Unfortunately, this system has a reduced effeciency at above 100 Gbaud and 16-QAM, and the nonlinear compensation becomes difficult to perform. The model [9] for the $1000 \mathrm{~km}$ wavelength-division multiplexing system with non-RZ and RZ modulation formats reveals a relatively low quality of data transmission and a significantly low signal power. The work [13] has presented a DWDM design with $25 \mathrm{~dB}$ reduction of the FWM power, which has limited value in choosing modulation formats with optical filters. The author [14] has proposed a DWDM design characterized by low distortion, high quality factor and low BER. It is less affected by the fibre nonlinearity, although the computational method is timeconsuming in performing iterative simulations. In Ref. [15], a hybrid DWDM with FWM suppression has been demonstrated. It has high enough gain and low input power, which have 
been achieved using circular polarization of light. This system shows that the light-polarization state also affects the system efficiency.

In summary, the problems of taking into account the FWM effect in the DWDM systems using the quality-of-transmission predictions and performing self-parametric optimization based on the field requirements need their further investigations along the direction of ML approaches. The understanding of FWM-influencing parameters would enhance the quality-of-transmission parameter and reduce the uncertainty for reconfigured DWDM systems applied in the future communications with high data rates. Therefore, it would be useful to explore the ML-based supervised parametric prediction models. Here the understanding of the FWM-influencing parameters and the corresponding mitigating factors, as well as achieving of appreciable reduction in the FWM, should be obtained using training databases of well established data points.

Of course, the optical DWDM networks of future generations with good performance and secured data transfer need larger number of channels, lower channel spacing and higher input power. These constraints imposed upon the DWDM system introduce challenging nonlinear impairments exemplified well by the FWM effect, which degrades the system performance and reduces both the bandwidth utilization and the channel capacity. Furthermore, these DWDM limitations require a complex design of the system structure and make the systems difficult to implement. In the present work, we discuss a SRM algorithm based on ML, which avoids the complexity peculiar for the conventional parametric simulation approaches. Moreover, we design a self-optimized high-capacity DWDM system operating at $2.56 \mathrm{Tbps}$, which offers real parametric trade-offs and a reduced FWM. Finally, we quantify our SRM-DWDM model by comparing its results with the exact results.

\section{Fundamentals of FWM}

Let us consider an optical system where light propagates at three different wavelengths, with the corresponding frequencies being equal to $F_{i}, F_{j}$ and $F_{k}$. Let an optical medium be characterized by a third-order susceptibility, which generates a fourth wave, of which frequency can be expressed as

$$
F_{i j k}=F_{i} \pm F_{j} \pm F_{k} \text {. }
$$

Here $F_{i j k}$ is the inter-modulated signal, or a FWM, which degrades the system function. Using a Taylor expansion, one can represent the combined fifth-order effects of the FWM as follows:

$$
\begin{aligned}
& B=B_{0}+\left(\Omega-\Omega_{0}\right) \frac{d \beta}{d \Omega}+\frac{1}{2}\left(\Omega-\Omega_{0}\right)^{2} \frac{d^{2} \beta}{d \Omega^{2}}+\frac{1}{6}\left(\Omega-\Omega_{0}\right)^{3} \frac{d^{3} \beta}{d \Omega^{3}}, \\
& +\frac{1}{d^{4}}\left(\Omega-\Omega_{0}\right)^{4} \frac{d^{4} \beta}{d \Omega^{4}}+\left(\Omega-\Omega_{0}\right)^{5} \frac{d^{5} \beta}{d \Omega^{5}}
\end{aligned}
$$

where $d \beta / d \Omega=\tau$ is the propagation delay per optical length, $\Omega-\Omega_{0}$ the frequency deviation, $1 / d^{4}$ the fourth-order dispersion factor, and $B_{0}$ the initial Taylor propagation factor. Since $\tau$ produces some phase delay, which does not influence the interference signal, it can be neglected. The phase-matching factor is defined as

$$
\Delta \beta=2 \pi^{2} \Delta f^{2}\left(\begin{array}{l}
\frac{\lambda^{2}}{2 \Delta c}+\frac{2 \pi \Delta f}{3} \frac{\lambda^{2}}{2 \Delta c^{2}}\left(\lambda^{2} \Delta_{1}+2 \lambda \Delta\right)+\frac{\pi^{2}}{3} \frac{\Delta f^{2} \lambda^{3}}{(2 \pi c)^{3}}\left(\lambda^{3} \Delta_{2}+6 \lambda^{2} \Delta_{1}+6 \lambda \Delta\right) \\
+\frac{2 \pi^{3}}{15} \frac{\Delta f^{3} \lambda^{4}}{(2 \pi c)^{4}}\left(\lambda^{4} \Delta_{3}+12 \lambda^{4} \Delta_{2}+36 \lambda^{4} \Delta_{1}+24 \lambda \Delta\right)
\end{array}\right),
$$

where $\lambda$ stands for the wavelength, $\Delta_{c}$ the chromatic dispersion in fibre, $\Delta f$ a function of channel 
spacing, $\Delta$ a function of delay, and $\Delta c$ a function of chromatic dispersion. Numerically, the FWM power can be easily expressed in terms of the input powers $p_{i}, p_{j}$ and $p_{k}$ at the frequencies $F_{i}$, $F_{j}$ and $F_{k}$, and the effective length of optical fibre:

$$
b_{F W M}=d_{i j k}^{2} v^{2} L_{\text {eff }}^{2} p_{i} p_{j} p_{k} \eta_{i j k} e^{-2 L},
$$

where $L$ denotes the length of fibre, $d_{i j k}$ is either the degeneration factor (at the condition $i=k$ ) or the non-degenerative factor (at $i \neq k$ ), $L_{\text {eff }}$ the effective length, $v$ the nonlinear coefficient, and $\eta_{i j k}$ the efficiency.

The nonlinear coefficient $v$ reads as

$$
v=\frac{2 \pi \eta_{2}}{A_{\text {eff }} \lambda_{0}},
$$

where $\eta_{2}$ is the refractive index of fibre core, $A_{\text {eff }}$ the effective area of fibre, and $\lambda_{0}$ the operating wavelength $(1550 \mathrm{~nm}$ in our case). The efficiency for the long-haul fibre due to FWM can be found from the relation

$$
\eta_{i j k}=\frac{\alpha^{2}}{\alpha^{2}+\Delta \eta B_{i j k}^{2}}\left(1+\frac{4 e^{-\alpha^{2}} \eta^{2}\left(\frac{\Delta B_{i j k}^{2}}{2}\right)}{\left(1-e^{-\alpha^{2}}\right)^{2}}\right),
$$

where $\alpha$ denotes the attenuation factor, $\Delta \eta$ the variation of refractive index and $B_{i j k}$ the phasematching factors at the frequencies numbered as $i, j$ and $k$. From the above relation, the product term with the exponential decay has no practical impact. So the simplified efficiency of the longhaul system is given by

$$
\eta_{i j k}=\frac{\alpha^{2}}{\alpha^{2}+\Delta \beta_{i j k}^{2}},
$$

where $\Delta \beta_{i j k}$ denotes the phase-matching coefficient. As specified by the International Telecommunication Union for the DWDM systems with equal channel spacing, $\Delta \beta_{i j k}$ takes discrete values, which are multiples of $0.4 \mathrm{~nm}$. Then the phase-matching coefficient can be written as

$$
\Delta \beta_{n}=n\left(\frac{d}{d v^{2}}\right) \Delta_{c} \Delta \lambda^{2} .
$$

Hence, the FWM efficiency depends upon the nonlinear refractive index $\eta_{2}$, the channel spacing $\Delta \lambda$, the dispersion coefficient $\delta$ and the effective area $A_{e f f}$ as follows:

$$
\eta_{i j k}=\frac{\eta_{2}}{A_{e f f}(\Delta \lambda) \delta} .
$$

Even the simplest analysis of the FWM testifies its importance and influence on the DWDM systems. This provides basic considerations of the need in mitigating the FWM for a conventional DWDM setup, provided that the DWDM functions such as channel spacing, optical gain, input power, data rate and other factors are known. Section 4 will explain the corresponding simulation setup, with detailed specification of all the procedures needed to perform the conventional simulation methodology. 


\section{Conventional simulation setup}

Fig. 1 shows a schematic diagram of a DWDM system. At a transmitter end of this system, a pseudo-random bit generator acts as a data source and the rate $10 \mathrm{Gbps}$ is used. The data of pseudo-random bit generator is converted into short electrical pulses through an electrical pulse generator, and a Mach-Zehnder modulator modulates these pulses. A DWDM multiplexer combines the input channels with the modulated optical signals and transmits them over a singlemode fibre having the dispersion $16.75 \mathrm{ps} / \mathrm{nm} / \mathrm{km}$ and the attenuation $0.2 \mathrm{~dB} / \mathrm{km}$. The optical signal from the single-mode fibre is transmitted through an EDFA amplifier. In this Section, we suppose that the amplification process is performed and the amplified signal is accumulated with a greater amplified spontaneous-emission noise. The amplified signal passes through the dispersioncompensating fibre characterized by some attenuation and dispersion parameters. Here nonlinear properties of the optical fibre and the dispersion-compensating fibre are actuated.

\section{DWDM Transmitter}

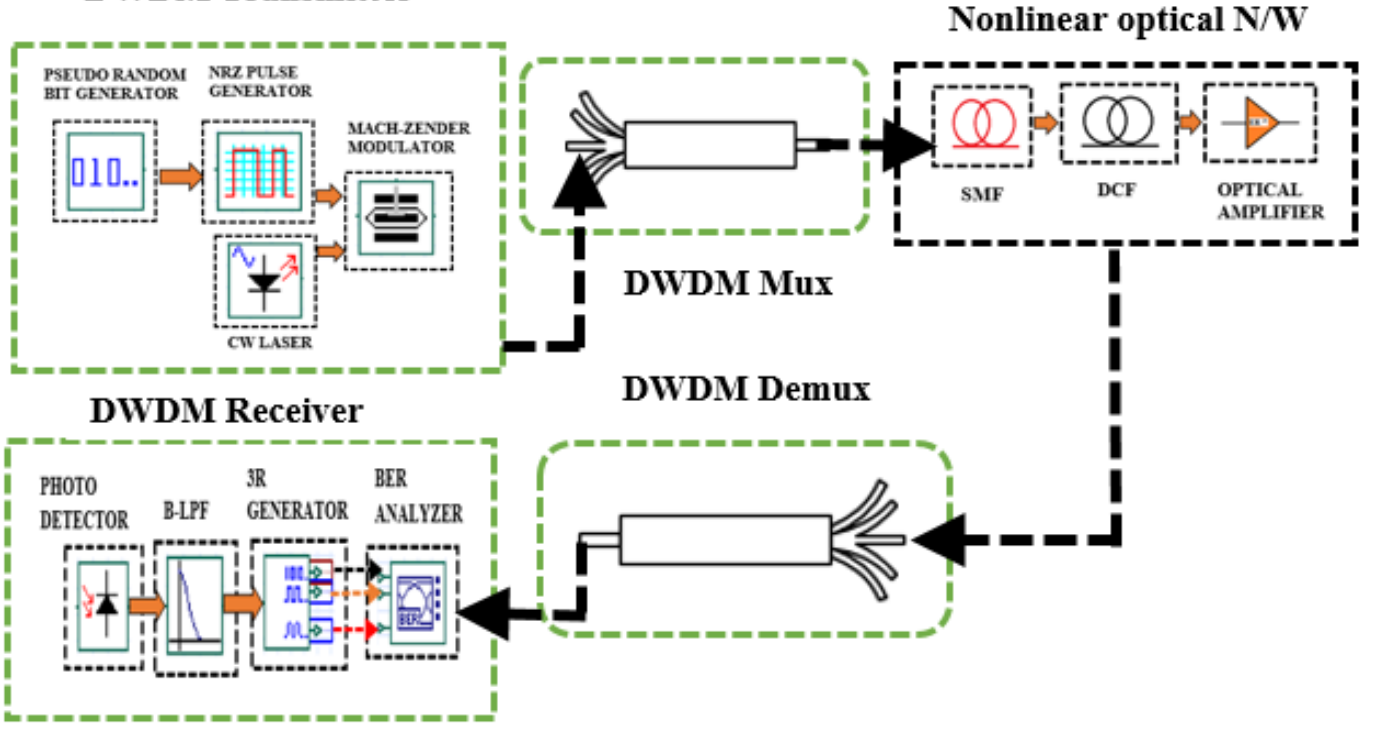

Fig. 1. A basic scheme of optical DWDM network: SMF is a single-mode fibre and DCF a dispersioncompensated fibre.

At a receiver side, a DWDM demultiplexer splits the demultiplexed signals into individual channels. The output signal emergent from demultiplexer passes through a PIN photodetector where conversion of optical signal into electrical one is performed. Then a converted electrical signal passes through a low-pass Bessel filter. Finally, the spectrum of the output signal in the form of a so-called 'eye characteristics' is detected by a BER analyzer.

Note that a high-power signal travels a long distance in a long-haul fibre-optic DWDM system to reach a receiver and it is cumbered by the nonlinearities. The simulated optical DWDM network must be designed with taking into account various system-dependent parameters, including the data rate, the channel spacing, the modulation format, the input power and the parameters of optical amplifier. In the presence of FWM, estimation of such factors as the minimum bit error rate, the Q-factor, the OSNR, the output signal power and the noise power is also necessary for any consistent analysis of DWDM performance. Analysis of the duty cycle, the optical gain and the effective area of fibre core is required, too. Section 5 presents the SRMDWDM model suggested by us and its methodology. The SRM is used for reducing the 
conventional iterative runtime. Moreover, it provides many details necessary for calculating the optimized network structure.

\section{SRM-DWDM system}

To mitigate the FWM effect, we implement the SRM via obtaining datasets from different analyzers such as optical spectrum analyzer, BER analyzer and wavelength-division multiplexing analyzer. Monitoring of these datasets demands different configurations of DWDM to achieve a near-field accuracy. However, retrieving of data from different monitoring points is difficult. Therefore, a reliable ML model which employs a regression-modelling method has to be developed in order to identify the main FWM-influencing parameters (e.g., the channel spacing, the input power, the data rate, the modulation format and the core size of fibre). An ML-based SRM control unit (or SRM-CU) collects the required data from a long-term network monitoring. Fig. 2 suggests possible approaches applied for collecting real data from the DWDM network. They are used while developing the SRM.

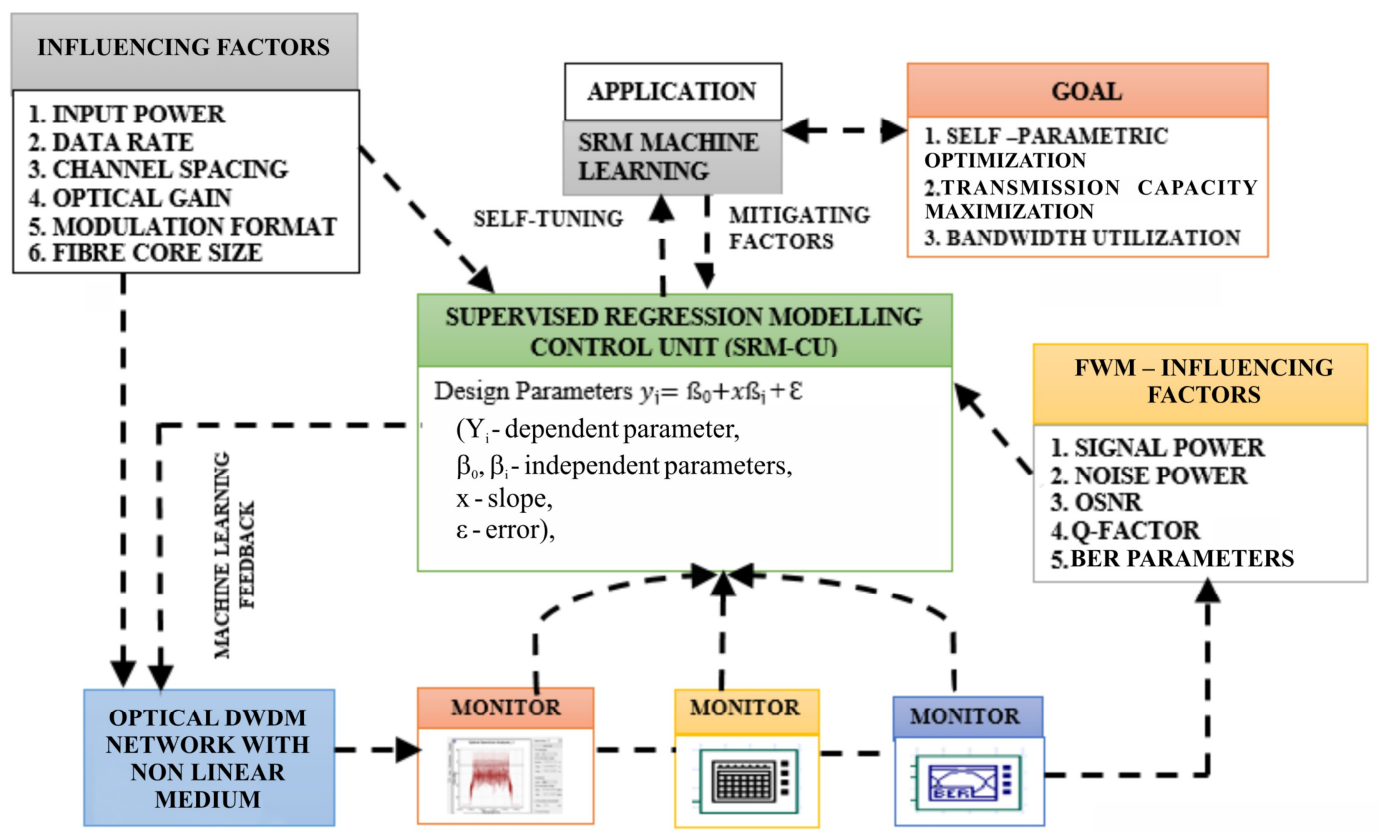

Fig. 2. A DWDM system with SRM suggested in the present work.

Fig. 2 illustrates functioning of our SRM-DWDM system (with a workflow used to retrieve the dataset and train the SRM), which performs a self-parametric optimization and mitigates the FWM. This setup analyzes the FWM-influencing parameters and the FWM-mitigating factors such as the maximal Q-factor (or simply Max Q-factor), the minimal BER (Min BER), the noise power and the OSNR from the long-term data-monitoring process. The appropriate aim is to develop the SRM and perform the self-parametric optimization. The SRM is built for different system functions and different factors that mitigate the FWM. Each factor is evaluated by a strength of correlation and a corresponding R-value.

Our SRM-DWDM system is designed basing on real-time constraints. This design is tuned via the FWM-influencing parameters: the input power, the data rate, the channel spacing, the modulation format and the optical gain. Then we analyze their influence on a high-capacity DWDM system. In this analysis, the FWM and its relationships with various influencing 
parameters are addressed. Their influence on bandwidth utilization and transmission capacity is modelled through the SRM-DWDM approach.

The SRM-DWDM system is studied using various conventional parametric simulation methods at different system functions and factors mitigating the FWM. The results are monitored with the wavelength-division multiplexing analyzer, the optical spectrum analyzer and the BER analyzer. The datasets are retrieved from the long-term network monitoring and fed into the SRMCU. The SRM-CU develops a knowledge database to perform the self-parametric optimization and collect much enough data for training our SRM-DWDM system. Finally, the SRM-CU is trained on different datasets from different configurations of the high-capacity DWDM system. The Rvalue obtained through the SRM shows the correlation level between the system functions and the mitigating factors. The R-values are also calculated in the SRM-CU.

Let us denote different values of the system variables (e.g., the input power, the channel spacing, the optical gain, the duty cycle and the bit rate) as $x$. In SRM-CU, the mitigating factors that influence the nonlinearities (e.g., the output signal power, the noise power, the OSNR, the eye height and the Max Q-factor) are considered as $y$ 's, with $\varepsilon$ being the error values. We collect $n$ such data points. Hence, our data points are $\left\{\left(x_{j}, y_{j}\right)\right.$, where $\left.j=1,2, \ldots \ldots n\right\}$. The supervised regression equation for the data is given by

$$
y_{j}=\beta_{0}+x \beta_{j}+\varepsilon,
$$

where $\beta_{0}$ 's are intersects and $\beta_{j}$ the slopes. By substituting the values obtained from simulations based upon Eq. (10), one can derive the SRM for different factors. Various iterative simulations are carried out by varying each of the system functions while keeping the others constant. The SRM-CU gets different values obtained from these factors, performs averaging and develops the SRM equation. The R-values achieved in this procedure show the correlations between different factors and a given system function. The levels of these correlations, which are distinguished by us, are shown in Table 1.

Table 1. R-values and their distinguished correlation levels.

\begin{tabular}{lll}
\hline$\#$ & R-value & Correlation level \\
\hline 1 & $1 \geq R \geq 0.8$ & High \\
2 & $0.7 \geq R \geq 0.5$ & Moderate \\
3 & $0.4 \geq R \geq 0.3$ & Low \\
4 & $0.2 \geq R \geq 0.1$ & Very low \\
5 & Negative $R$ value & No \\
\hline
\end{tabular}

The above calculations have been performed for the training dataset received from the SRMCU. For example, one of the equations obtained for the 1.28 Tbps DWDM network configuration is as follows:

$$
\text { output signal power }=[-0.4729] \times[\text { bit rate }]+[10.2464] \text {, }
$$

with the R-value being equal to 0.9362 . From the above study, the correlation between the output signal power and the input power can be highlighted by the R-value. The figure 0.9362 demonstrates that the correlation between the input power and the output signal power is strong. On the other hand, we have

$$
\text { OSNR }=[-0.2555] \times[\text { bit rate }]+[51.4391]
$$


with the R-value $=0.4269$. This proves that there is a low correlation between the input power and the OSNR.

In Section 6, we will compare the estimated parameters of our SRM-DWDM model with their true values, in particular for the Q-factor, the noise power, the OSNR, the signal power and the eye height. Then the accuracy of the SRM-DWDM model can be estimated by a difference between the estimated and true values, which is treated as an error.

\section{Testing of SRM-DWDM system and parametric observations}

The self-parametric optimization is performed through a trained knowledge database acquired from the SRM-CU block mentioned in Fig. 2. Prioritization of the nonlinear effects occurring in the DWDM system enhances its performance and reduces its complexity. Our model is developed basing on the SRM, which provides the correlation levels for the relationships among the FWMmitigating factors and the system functions (e.g., the channel spacing, the input power, the bit rate, the modulation format, the core size of fibre and the optical gain). Notice that high-capacity DWDM systems with higher-order modulations are more sensitive to the FWM. Our model can also be used for the higher-order modulated DWDM systems, thus providing basic understanding of the FWM-influencing factors. Here the correlation level of a given mitigating factor shows how much this factor can influence a given system function. This helps in building dynamically a selfoptimized system using the SRM-DWDM approach. The corresponding equations developed from the SRM-DWDM are used to find the correlations of different factors with the system functions.

We have developed the supervised regression models for the particular cases of $1.28 \mathrm{Tbps}$ and 2.56 Tbps data rates. Since the efficient high-capacity DWDM systems must involve a high input power, a large number of channels, low channel spacing and a low-distortion modulation format, we have modelled our DWDM as being operated at the data rate $10 \mathrm{Gbps}$, the narrow channel spacing $(50 \mathrm{GHz})$, the input power $10 \mathrm{dBm}$, the fibre-core size $80 \mu \mathrm{m}$ and the RZ modulation format with the duty cycle $0.25 \%$. To perform the SRM, every of these parameters is varied while keeping the other parameters invariable. The conventional iterative results, i.e. the true results, are gathered with the simulations performed via OptiSystem. These true iterative results are considered as a trained database. They we feed the SRM-CU with these values to train it. The number $n$ of those iterations is large enough.

Table 2. Conventional iteration results obtained for the $1.28 \mathrm{Tbps}$ system at different bit rates.

\begin{tabular}{lllllll}
\hline $\begin{array}{l}\text { Bit } \\
\text { rate }\end{array}$ & $\begin{array}{l}\text { Signal } \\
\text { power, } \mathrm{dBm}\end{array}$ & $\begin{array}{l}\text { Noise power, } \\
\mathrm{dBm}\end{array}$ & OSNR, dB & Max Q-factor & Min BER & Eye height \\
\hline 2.5 & 9.339 & -42.314 & 51.654 & 29.743 & $2.27 \mathrm{E}-195$ & 0.00271 \\
5.0 & 7.864 & -42.218 & 50.082 & 64.760 & 0 & 0.00207 \\
7.5 & 5.910 & -41.211 & 47.121 & 71.760 & 0 & 0.000756 \\
10.0 & 6.050 & -44.462 & 50.512 & 77.761 & 0 & 0.000924 \\
\hline
\end{tabular}

The regression-model equations have been built for our SRM-DWDM system. So, when the input power is $10 \mathrm{dBm}$ in the $1.28 \mathrm{Tbps}$ system, we obtain

$$
\text { output signal power }=[0.999] \times[\text { input power }]+[16.271],
$$

with the R-value 1.000. From Table 2, the bit rate and the output signal power are correlated. Issuing from the R-value, the relationship between the input power and the output signal power is significant. The 'true' value $26.231 \mathrm{dBm}$ and the estimated value $26.261 \mathrm{dBm}$ are obtained for this mitigating factor, with a low marginal error of 0.0298. Similarly, for the other FWM-mitigating 
factor in the same DWDM configuration and the input power $10 \mathrm{dBm}$ we have

$$
\text { eye height }=[0.0011] \times[\text { input power }]+[0.0064] \text {, }
$$

with the R-value 0.0865. It is seen from Table 2 that the bit rate and the other FWM-mitigating factors such as the output signal power and the eye height are also correlated. There is very weak relationship between the input power and the eye height, since the R-value is very low. Furthermore, wide marginal errors are obtained for the true $(0.0263)$ and estimated $(0.0174)$ values of the eye-height factor. In other words, the true and estimated values of the eye-height factor reveal high marginal deviations. The similar results can be observed with the probability plots for the eye-height characteristics. Obviously, much larger numbers of trained datasets are needed to achieve higher accuracy and lower marginal errors for the eye-height factor.

In a quite similar manner, the regression-model equations have been developed for the other combinations of the influencing parameters to understand their effects on the FWM. This is necessary for structuring well our high-capacity SRM-DWDM so that it met the global optimization requirements.

Table 3. Mean, standard deviation, P-value and Anderson-Darling factor obtained for the parameters that influence the FWM nonlinearity, using our SRM-DWDM system.

\begin{tabular}{lcccc}
\hline Parameter & Mean & Standard deviation & $\begin{array}{c}\text { Anderson-Darling } \\
\text { factor }\end{array}$ & P-value \\
\hline \multirow{2}{*}{ Signal power, dBm } & 17.11 & 10.68 & 0.367 & 0.371 \\
& 16.88 & 10.83 & 0.400 & 0.306 \\
\hline \multirow{2}{*}{ Noise power, dBm } & -33.26 & 12.22 & 0.298 & 0.63 \\
& -33.02 & 11.43 & 0.477 & 0.193 \\
\hline \multirow{2}{*}{ Q-factor } & 40.6 & 24.43 & 1.778 & $<0.005$ \\
& 41.31 & 24.49 & 1.677 & $<0.005$ \\
\hline \multirow{2}{*}{ OSNR, dB } & 50.71 & 8.653 & 1.700 & $<0.005$ \\
& 49.78 & 8.452 & 1.216 & $<0.005$ \\
\hline \multirow{2}{*}{ Eye height } & 0.0104 & 0.0121 & 1.605 & $<0.005$ \\
& 0.0229 & 0.0554 & 2.909 & $<0.005$ \\
\hline
\end{tabular}

The SRM equations applied in different ranges of the bit rate and the appropriate results predicted for different mitigating factors in the 1.28 and $2.56 \mathrm{Tbps}$ DWDM configurations are qualitatively the same. The SRM-based estimated value in this case has also been verified after simulating the same parameter, which is given as an input to the regression equation. Following from the SRM-CU knowledge database, we conclude that the Q-factor, the signal power, the noise power and the OSNR manifest very low marginal error differences between the estimated and true values. On the contrary, the estimated values of the eye height show more deviations when compared to the true values observed from the conventional iterations. To reduce this error difference, a more trained dataset for the eye height must have been analyzed. In general, validation process has yielded in very similar sets of results in the both calculations, which demonstrates a remarkable accuracy of global optimization and offers real trade-offs among userbased requirements.

The error difference parameters derived as explained above can be fitted well by the normal probability distribution. Table 3 presents the mean and the standard deviation for both the true and 
estimated values in our SRM-DWDM system, as obtained for the cases of 1.28 and 2.56 Tbps bitrate fibre-optic systems.

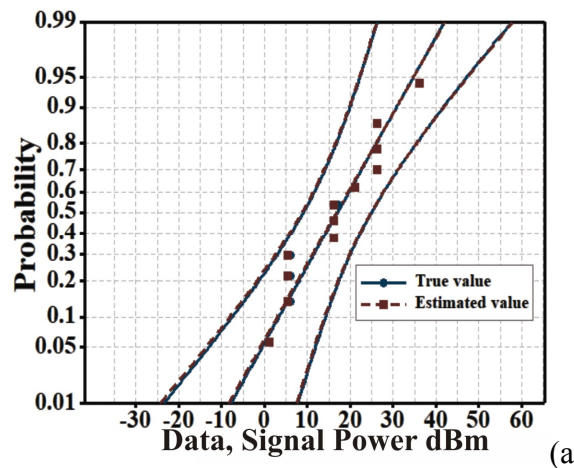

(a)

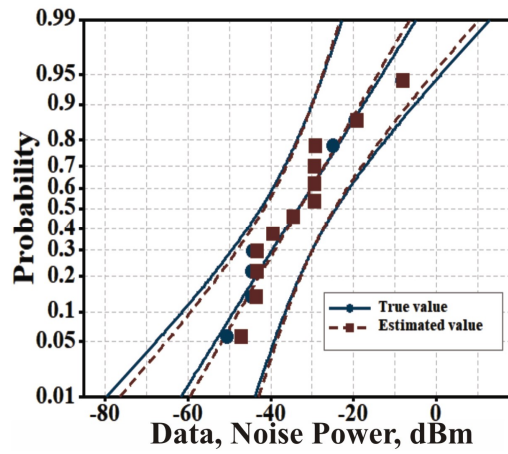

(b)
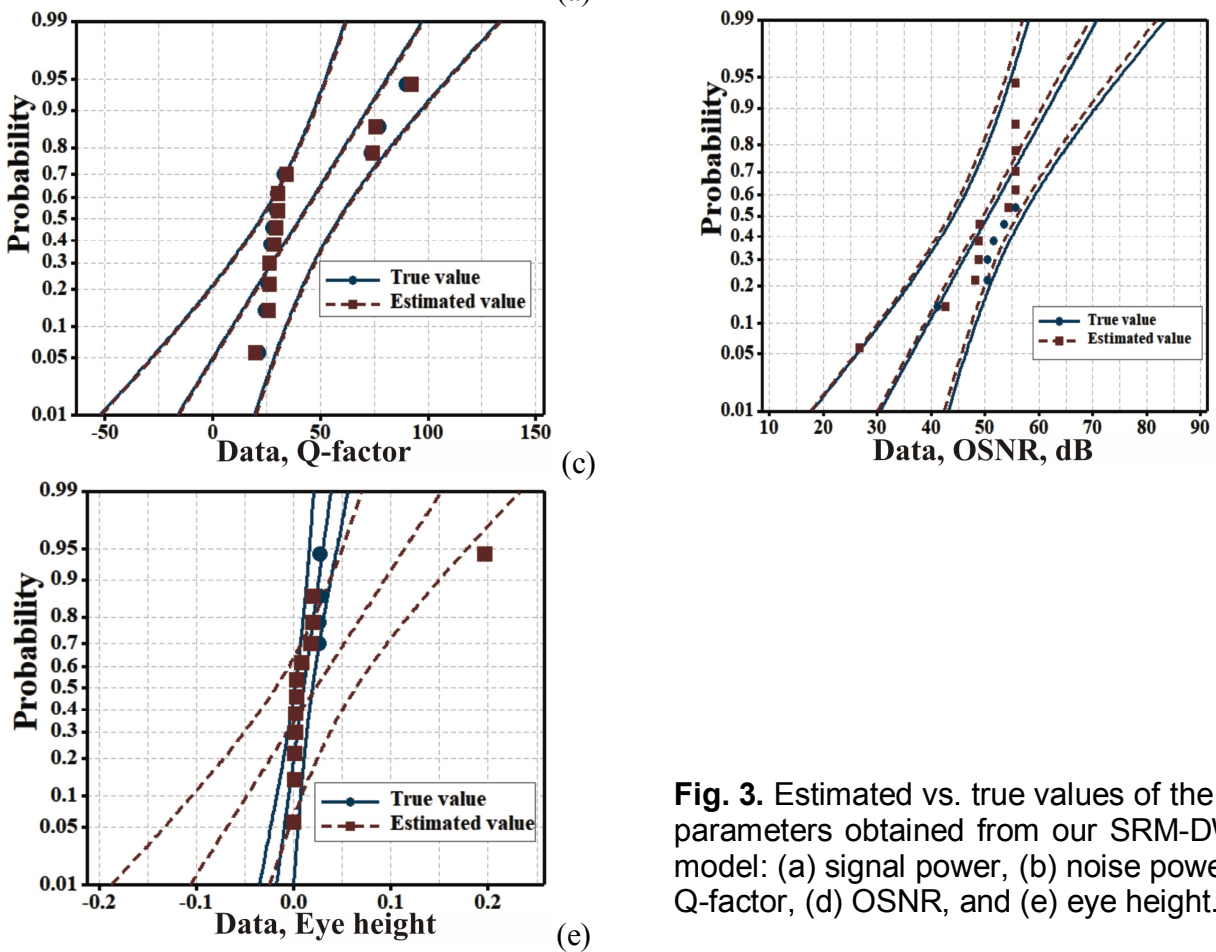

(e)

Fig. 3. Estimated vs. true values of the main parameters obtained from our SRM-DWDM model: (a) signal power, (b) noise power, (c) Q-factor, (d) OSNR, and (e) eye height.

Fig. 3 shows the normal probability plots for the FWM-mitigating factors (the signal power, the noise power, the Q-factor, the OSNR and the eye height), which have been obtained from the longterm network monitoring and the SRM-DWDM modelling. It is evident that, except for the eye height, all the remaining factors follow closely the ideal normal distribution. The Anderson-Darling method receives the dataset from the SRM-CU, checks the flow of estimated values following the true values and analyzes the distribution. The estimated values of the signal power, the noise power, the Q-factor and the OSNR in the plots fall on straight lines as functions of the true values. Fig. 3 testifies that the SRM-DWDM-based distributions are close to the actual underlying distribution. Except for the eye height, the distribution plots obtained for all the other parameters reveal no enough evidence for extra deviations from the normal distribution. Unlike all the other parameters, the deviations for the eye-height characteristic expressed in terms of the mean and standard deviation are severe and do not follow the normal distribution (see Table 3). Further investigations must be carried out to understand better the eye-height characteristic and reduce the error margins. 


\subsection{Effects of bit rate}

Fig. 4 displays the spectral data obtained for the 256-channel network operating at different bit rates, using our SRM-DWDM system. In this setup, the bit rate varies from 2.5 to $10 \mathrm{Gbps}$ and the remaining system parameters are kept constant.

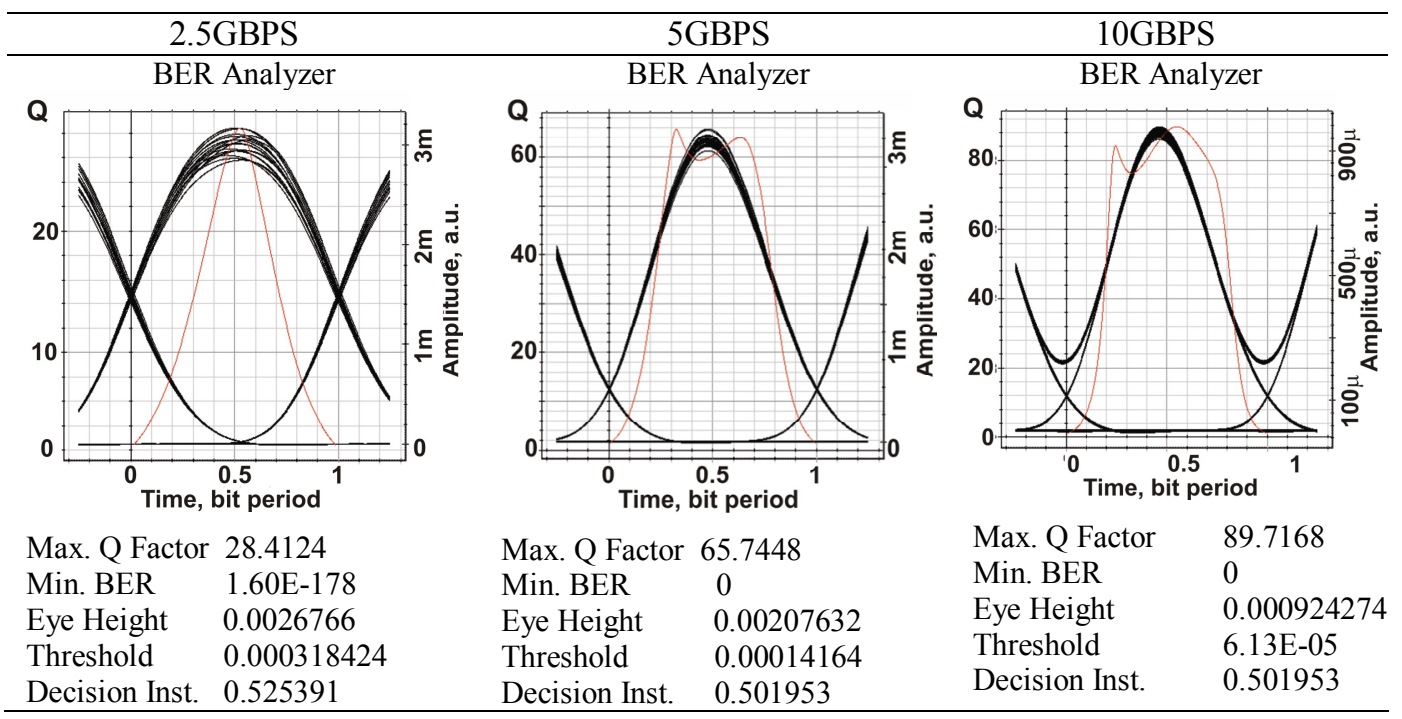

Fig. 4. Spectral analysis data obtained for 2.56 Tbps DWDM system at different bit rates.

One can see from this simulation results that the output signal power, the Q-factor, the BER and the eye diagram have a direct impact on the FWM nonlinearity, which degrades the performance of optical DWDM systems. Increasing the data rate stipulates higher dispersion which introduces pulse broadening and creates more noise interference in the DWDM system. As seen from the eye characteristic shown in Fig. 4, the output spectral characteristic at $10 \mathrm{Gbps}$ shows low eye height $(\sim 0.0009)$ and high enough pulse broadening in the output signal. Such factors as the output signal power, the noise power and the OSNR also have their impact due to occurrence of dispersion in the DWDM system, which affects the Q-factor. Simulations for the SRM-based data obtained for the 128- and 256-channel capacities testify that the input power is highly correlated with the output signal power, the eye height and the Max Q-factor, which is seen from the corresponding R-values.

\subsection{Effects of channel spacing}

In this section we study SRM-DWDM results obtained at the channel spacings varying from 25 to $100 \mathrm{GHz}$ in case of the 128- and 256-channel configurations. The channel spacing is a key simulated parameter, which is inversely related to the system functions that mitigate the FWM in a fibre-optic DWDM system. After analyzing the datasets from different configurations, one can conclude that the system with 128 channels utilizes the channel spacing very well if compared to the case of 256 channels. It is seen from Fig. 5 that the system with narrow channel spacing $(25 \mathrm{GHz})$ has a greater noise interference, which imposes a distorted eye height and a poor Qfactor. The system with $100 \mathrm{GHz}$ channel spacing shows a very good eye height, a high Q-factor, a low BER and a very efficient eye characteristic. The OSNR and the Q-factor are closely correlated and have a positive impact, irrespective of the channel spacing. The channel spacing is a real trade-off factor, which needs to be optimized basing on user requirements (e.g., large number of

Ukr. J. Phys. Opt. 2021, Volume 22, Issue 1 
channels and extremely good bandwidth utilization). Finally, our SRM-DWDM technique reveals that increasing channel spacing lowers the FWM and provides a sufficient Q-factor with a very good eye height.

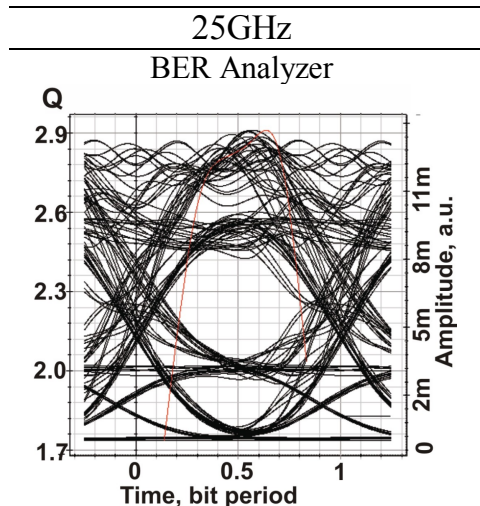

Max. Q Factor

Min. BER

Eye Height

Threshold

Decision Inst.
2.90984

0.00180202

$-0.00027415$

0.00594101

0.636719

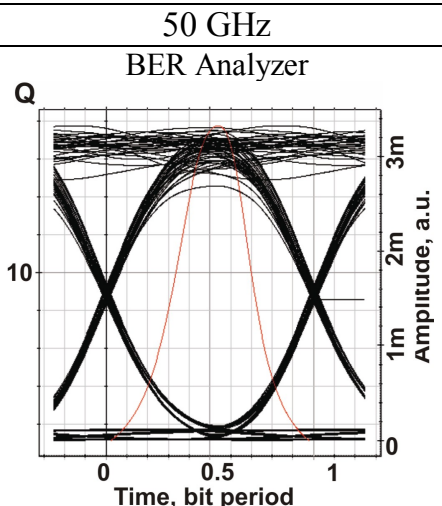

Max. Q Factor

Min. BER

Eye Height

Threshold Decision Inst.
17.7373

9.74E-71

0.00249052

0.000918246 0.539063

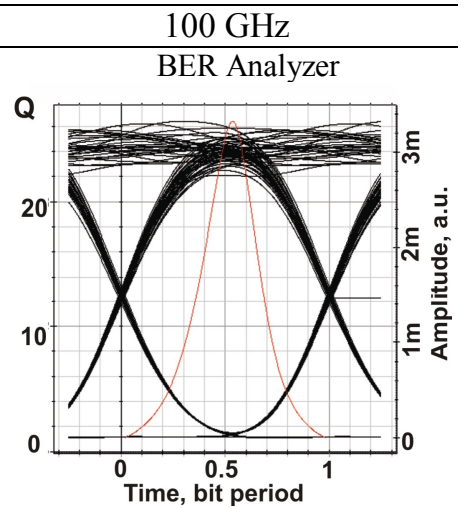

Max. Q Factor 26.405

Min. BER 4.37E-154

Eye Height $\quad 0.00264921$

Threshold $\quad 0.000410038$

Decision Inst. 0.535156

Fig. 5. Spectral analysis data obtained for a 1.28 Tbps DWDM system at different channel spacings.

\subsection{Effects of $R Z$ modulation at different duty cycles}

Fig. 6 displays the spectral characteristics obtained by our SRM-DWDM system at the RZ modulation and different duty cycles (from 0.25 to 0.50 ). They all refer to the 256-channel capacity. In long-haul DWDM data transmission, the RZ and NRZ modulation formats are widely used. The RZ format is a key method used up to $2.56 \mathrm{Tbps}$, since it is characterized by higher OSNR and peak power and lower BER than those typical for the other modulation formats. It is also known that this format provides good immunity against the nonlinear effects in fibre-optic systems and so is capable of mitigating the FWM effect.

Our SRM-based modelling has provided the R-values and the corresponding correlation levels for the Q-factor, the BER, the output signal power, the OSNR and the noise power. The noise power reveals a moderate correlation, with the R-value equal to $\sim 0.678$. The $\mathrm{Q}$-factor and the eye height show higher correlations at the RZ modulation and varying duty cycle, which proves its high immunity against the FWM nonlinearities. As a consequence, the results of our SRM-DWDM technique testify the fact of sufficient data transmission level, with high Q-factor, low BER and good eye height.

One can see from Fig. 6 that the RZ modulation format with the duty cycle 0.3 has a good Qfactor $(\sim 36.05)$, the eye height $8.86 \times 10^{-5}$ and a narrow output pulse. The other duty cycles reveal the occurrence of broadened output-pulse characteristics in the presence of nonlinearities. These results are also directly reflected in the other FWM-mitigating factors such as Min BER, the OSNR, the Q-factor, the output signal power and the noise power. This drops down the system performance. One can conclude from the SRM analysis that the system with the RZ modulation and variable duty cycle behaves better than the other compensation schemes. We observe marginal correlations for the eye height, the output signal power and the noise power, which influence the Q-factor and the parameter Min BER identically. 


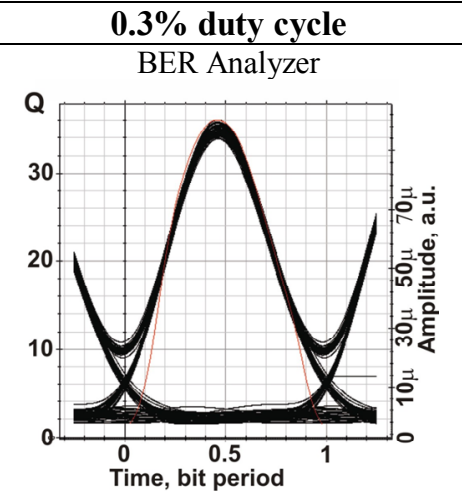

Max. Q Factor

Min. BER

Eye Height

Threshold

Decision Inst.
36.0525

$6.19 \mathrm{E}-285$

$8.86 \mathrm{E}-05$

$3.90 \mathrm{E}-05$

0.457031

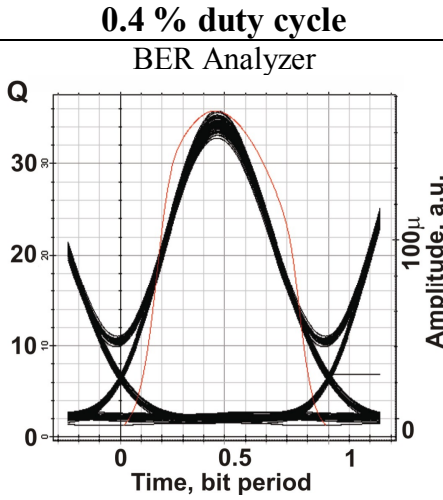

Max. Q Factor

Min. BER

Eye Height

Threshold

Decision Inst.
35.7236

7.66E- 280

0.000150265

4.44E-05

0.453125

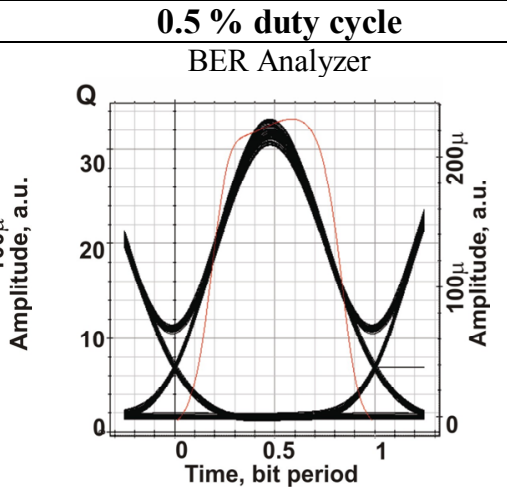

Max. Q Factor 33.2279

Min. BER 1.76E-242

Eye Height $\quad 0.000199907$

Threshold 4.02E-05

Decision Inst. 0.585938

Fig. 6. Spectral analysis data obtained for a 2.56 Tbps DWDM system at RZ modulation format and different duty cycles.

\subsection{Effects of input power}

The input power is the most investigated and highly correlated system parameter that directly affects all the factors influencing the FWM nonlinearities in fibre-optic DWDM systems. Fig. 7 shows the spectral characteristics obtained with our SRM-DWDM system at different input powers (from 10 to $-10 \mathrm{dBm}$ ). The resultant spectral characteristics confirm that variations in the input power affect directly the FWM nonlinearities in the DWDM system. This phenomenon is proven by the correlation analysis, which shows that the output signal power, the noise power, the Q-factor, the BER and the eye diagram are highly correlated with the input power. In other words, any variations in this power would influence hard all of these parameters and make high impact on the nonlinearity. It would be useful to find the optimized input power to transmit data efficiently over long distances.

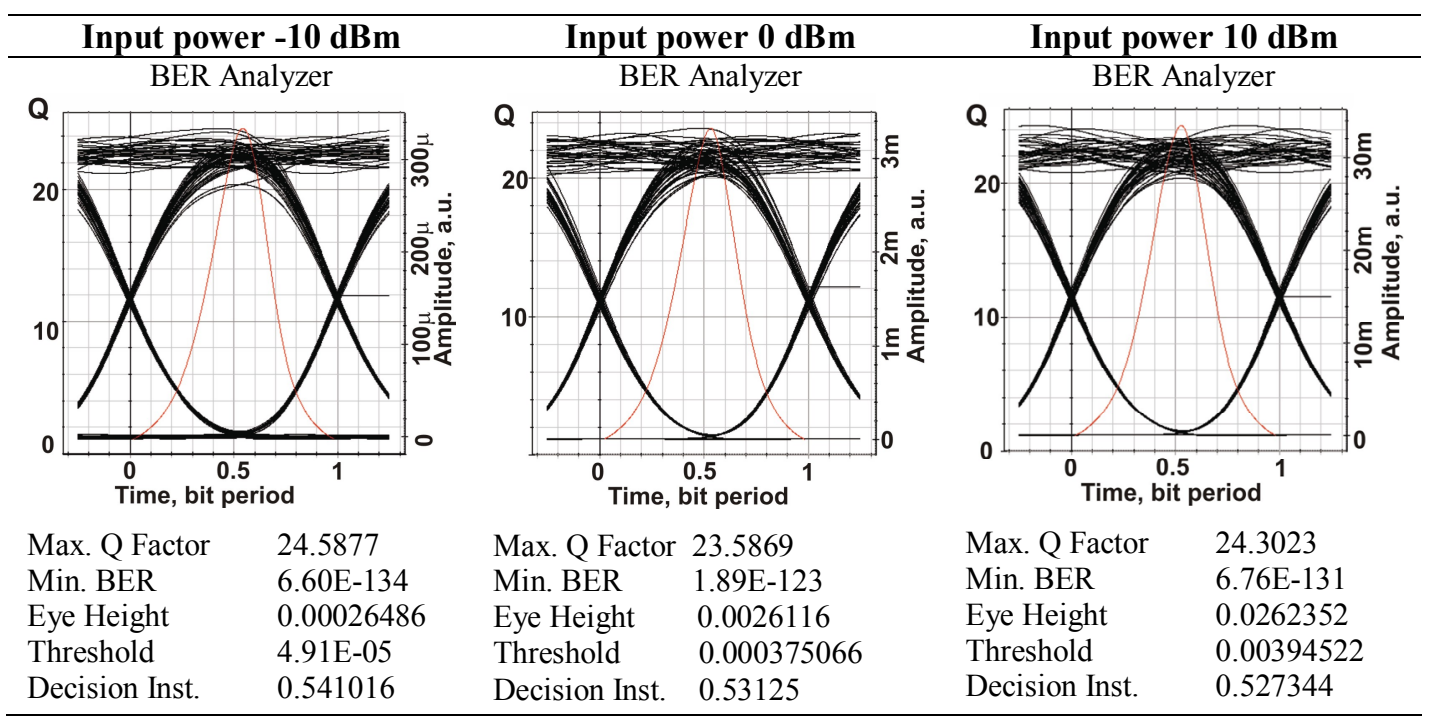

Fig. 7. Spectral analysis data obtained for a 1.28 Tbps DWDM system at different input powers. 
In general, the optimal input power varies depending on the transmission distance and the other system parameters. It is evident from Fig. 7 that, for a 128-channel system, the OSNR decreases with decreasing input power. One has the BER $6.6 \times 10^{-134}$, the eye height $\sim 0.0003$ and the maximum quality factor $\sim 24.6$, which are larger than those obtained for a 256-channel system. The input power is linearly related to the output signal power, the noise power, the Q-factor and the eye height. The SRM-DWDM results associated with high R-values and good correlations among the output signal power, the noise power, the Q-factor and the eye height prove that any deviations in the input power influence notably the FWM, which can degrade the system parameters.

\subsection{Effects of core size of optical fibre}

The effective area of the fibre-core size influences the FWM. Namely, increasing core size reduces proportionally the effective core area and so results in diminishing the FWM. In our study, the effective core radius varies from 80 to $160 \mu \mathrm{m}$. The appropriate simulation results are presented in Fig. 8. One can see that the effect of FWM is essential for large numbers of channels and high data rates, which lowers the system performance. This is clearly visible from our SRM-based study, which demonstrates that increasing effective core radius reduces the FWM.

BER Analyzer
Max. Q Factor 24.4452
Min. BER
Eye Height
Threshold
Decision Inst.
$0.00 \mathrm{E}-132$

Fig. 8. Spectral analysis data obtained for a 2.56 Tbps DWDM system at different core sizes of optical fibre.

Our SRM-based results derived from the statistical data obtained by simulating the DWDM system at different core sizes show that a variable input power is directly related to the noise power. Then the increasing noise power influences the remaining parameters so that the complete system performance degrades.

\subsection{Effects of optical gain}

Our last simulations refer to the SRM-DWDM data obtained at varying gain of optical amplifier, under condition that the rest of the parameters are constant. The SRM analysis reveals high enough R-values for the output signal power, the noise power, the Q-factor and the eye diagram. Again, we have good enough spectral characteristics and mitigation of the FWM effect. In other words, such nonlinearity-influencing factors as the output signal power, the noise power, the Q-factor and the eye diagram are highly subjected to changes in the optical-amplifier gain. 
The SRM-DWDM data at high optical gains implies high output power, along with high FWM power. The simulation results also reveal a better performance of the 256-channel system, which has a small improvement in the BER $\left(5.03 \times 10^{-163}\right)$, the eye height $\sim 0.0267$ and the Qfactor 27.172, whereas the rest of the outputs remain similar, except for the OSNR and the output signal power which become slightly improved. Our SRM-DWDM analysis yields in high Rvalues, which shows strong correlations of all the parameters such as the output signal power, the noise power, the Q-factor and the eye characteristic.

20dB
Max. Q Factor 25.2275
Min. BER
Eye Height
Threshold
Decision Inst.
7.78E-141

Fig. 9. Spectral analysis data obtained for a 2.56 Tbps DWDM system at different optical gains.

\section{Conclusions}

In the present work, the self-parametric optimization of the high-capacity DWDM system is performed through the ML-based SRM scheme under a complicated condition of FWM effect present. According to our approach, the SRM-CU analyzes the dataset from the trained knowledgebase and provides the R-value. Then the calculations are performed for different combinations of the FWM-influencing parameters. We achieve a high enough accuracy to meet the requirements for trade-off among such factors as the maximal Q-factor, the minimal BER, the output power and the OSNR, in order to mitigate the FWM and cross-talks in the DWDM system. In our SRM-DWDM method, the estimated values of the eye height deviate significantly from the true values. Further investigations are needed to understand the eye-height characteristic and reduce the corresponding error margins. In general, our SRM-DWDM approach makes the DWDM design more reliable, easy and efficient. As a consequence, this can ensure higher quality of data transmission over longer distances.

Hence, we declare a consistent design of high-capacity DWDM systems and solving a complex problem of FWM nonlinearity, using the SRM-based analytical models. It also enables deriving some input-output relations among the system functions and its desired output parameters. The SRM can ensure extensive studies of the parameters and the factors that mitigate the FWM effect. The SRM technique applied to high-capacity DWDM systems enables one to understand and classify the data automatically and associate a streamline analysis for meeting complex system-design requirements, thereby reducing the system complexity and the computational runtime. A clear comparison of the estimated and true (iterative) results for any 
DWDM system provides a firm ground for both evaluation and verification of the SRM-DWDM approach, which is based upon calculations of the accuracy levels through the marginal errors.

\section{Acknowledgement}

We thank Prof Dr. N. R. Shanker, R\&D Manager at Chase Technologies, for useful discussions about the present study.

\section{References}

1. Ahmed J, Hussain A, Siyal M Y, Manzoor H and Masood A, 2014. Parametric analysis of four wave mixing in DWDM systems. Optik. 125: 1853-1859.

2. Udalcovs A, Schatz R, Wosinska L and Monti P, 2017. Analysis of spectral and energy efficiency tradeoff in single-line rate WDM links. J. Light. Technol. 35: 1847-1857.

3. Kathpal N and Garg A, 2020. Analysis of radio over fiber system for mitigating four-wave mixing effect. Dig. Commun.Net. 6: 115-122.

4. Nguyen B, Nguyen H T, Van Nguyen D, Van Nguyen T and Nguyen Q T, 2019. Impact of frequency shift on nonlinear compensation using optical phase conjugation for M-QAM signals. Rev. J. Electron. Commun. 9: 55-62.

5. Sabapathi T and Poovitha R, 2019. Mitigation of nonlinearities in fiber optic DWDM system. Optik. 185: 657-664.

6. Huszaník T, Turán $\mathrm{J}$ and Ovsenik L, 2019. On mitigation of four-wave mixing in high capacity ultra-DWDM system. $20^{\text {th }}$ International Carpathian Control Conference (ICCC), Krakow-Wieliczka, Poland, pp. 1-4.

7. Habib Ullah Manzoor, Tareq Manzoor, Ashiq Hussain and Moustafa H Aly, 2020. FWM mitigation in DWDM optical networks. J. Phys.: Conf. Series. 1447: 012033.

8. Kaler R and Kaler R S, 2012. Investigation of four wave mixing effect at different channel spacing. Optik. 123: 352-356.

9. Ajmani Manisha, Singh Preeti and Kaur Pardeep, 2019. Hybrid dispersion compensating modules: A better solution for mitigating four-wave mixing effects. Wireless Personal Commun. 107: 959-971.

10. Sharma V and Kaur R, 2013. Implementation of DWDM system in the presence of four wave mixing (FWM) under the impact of channel spacing. Optik. 124: 3112-3114.

11. Rashed A, 2019. Comparison between NRZ/RZ modulation techniques for upgrading long haul optical wireless communication systems. J. Opt. Commun. 10.1515/joc-2019-0038 (article in press).

12. Meena D, Sarath K T, Francis F, Dipin E and Srinivas T, 2019. Mitigation of EDFA transient effects in variable duty cycle pulsed signals. Def. Techn. 15: 276-281.

13. Ahmed J, Hussain A, Adeel F, Siyal M Y and Yu C, 2013. Optical signal processing using four wave mixing in highly nonlinear silicon. Optik. 124: 3439-3442.

14. Habib Ullah Manzoor, Tareq Manzoor, Ashiq Hussain, Moustafa H Aly and Sanaullah Manzoor, 2019. FWM reduction using different modulation techniques and optical filters in DWDM optical communication systems: A comparative study. Iran. J. Sci. Technol. Trans. Electr. Eng. 43: 479-488.

15. Hamadamin J A, Ibrahim M A and Saleem S M, 2020. Design and performance optimization of WDM lightwave system using optimum modulation formats. Nat. Acad. Sci. Lett. 43: 523-52.

16. Kumar Ghanendra and Kumar Sandeep. 2019. Investigation of the performance of optical 
amplifiers for a $96 \times 12$ Gbps DWDM system using ultrasmall channel spacing. Photon. Network Commun. 38: 108-114.

17. Obaid Hafiz and Shahid Hifsa, 2019. Performance evaluation of hybrid optical amplifiers for a $100 \times 10$ Gbps DWDM system with ultrasmall channel spacing. Optik. 200: 163404 .

18. Ji Y, Zhang J, Wang X and Yu H, 2018. Towards converged, collaborative and co-automatic (3C) optical networks. Sci. China Inf. Sci. 61: 1-19.

19. Ghazisaeidi A, 2019. Theory of coherent WDM systems using in-line semiconductor optical amplifiers. J. Light. Technol. 37: 4188-4200.

20. Gu R, Yang $\mathrm{Z}$ and Ji Y, 2020. Machine learning for intelligent optical networks: A comprehensive survey. J. Netw. Comput. Appl. 157: 102576.

21. Musumeci F, Rottondi C, Corani G, Shahkarami S, Cugini F and Tornatore M, 2019. A tutorial on machine learning for failure management in optical networks. J. Light. Technol. 37: 4125-4139.

22. Seve E, Pesic J, Delezoide C, Bigo S and Pointurier Y, 2018. Learning process for reducing uncertainties on network parameters and design margins. J. Opt. Commun. Net. 10: A298A306.

23. Junfeng Xie, F Richard Yu, Tao Huang, Renchao Xie, Jiang Liu, Chenmeng Wang and Yunjie Liu 2019. A survey of machine learning techniques applied to software defined networking (SDN): Research issues and challenges. IEEE Commun. Surv. Tutorials. 21: 393-430.

24. Zeadally $S$ and Tsikerdekis M, 2020. Securing Internet of Things (IoT) with machine learning," Int. J. Commun. Syst. 33: 1-16.

25. Alsheikh M A, Lin S, Niyato D and Tan H P, 2014. Machine learning in wireless sensor networks: Algorithms, strategies, and applications. IEEE Commun. Surv. Tutorials, 16: 19962018.

26. Bhatti D M S, Ahmed S, Chan A S and Saleem K, 2020. Clustering formation in cognitive radio networks using machine learning. AEU - Int. J. Electron. Commun. 114: 152994.

27. Mao Q, Hu F and Hao Q, 2018. Deep learning for intelligent wireless networks: A comprehensive survey. IEEE Commun. Surv. Tutorials. 20: 2595-2621.

28. Danish Rafique and Luis Velasco, 2018. Machine learning for network automation: Overview. Architecture, and Applications [Invited Tutorial]. J. Opt. Commun. Net. 10: 126-143.

29. Pacheco F, Exposito E, Gineste M, Baudoin C and Aguilar J, 2018. Towards the deployment of machine learning solutions in network traffic classification: A systematic survey. IEEE Commun. Surv. Tutorials. 21: 1988-2014.

Venkatesan K., Chandrasekar A. and Ramesh P. G. V. 2021 Supervised regression modelling for mitigation of four-wave mixing in dense wavelength-division multiplexing systems. Ukr.J.Phys.Opt. 22: 12 - 30. doi: 10.3116/16091833/22/1/12/2021

Анотація. Нещодавня глобальна криза, пов'язана з COVID-19, спонукала мільйони людей прачювати вдома, що спричинило різке збільшення загального мережевого трафіку, вимог до швидкості передачі даних та кіниевих можливостей мережі. Це також спричинило більше шумів, перехресних завад і небажаних нелінійностей оптичного волокна, особливо ефекту чотирихвильового змімування (ЧХЗ), який погіршуе роботу систем мультиплексування зі щұільним поділом довжини хвилі (МЩПХ). Наявність ЧХЗ у системах МЩПХ спонукає зростаючу складність та інериійність мереж $i$ знижуе ӥхню спектральну ефективність. У свою чергу, ие погіршує ефективне використання оптичної смуги

Ukr. J. Phys. Opt. 2021, Volume 22, Issue 1 
пропускання. Щоби пом'якшити зазначені проблеми, ми пропонуємо регресійне моделювання з учителем (РМУ). Відповідний підхід РМУ-МЩПХ здійснює автоматизовану оптимізацію систем МЩПХ за допомогою методів машинного навчання $i$ знаходить реальні компроміси серед різних факторів, щчо впливають на ЧХЗ. Наша модель понижує складність системи та час обчислень, щзо приводить до точного та надійного прогнозування значень параметрів. Ми також оцінили ефективність методики РМУМЩПХ, порівнюючи ї̈ дані з результатами, одержаними ітераційними методами для різних параметрів (наприклад, для відношення вихідного сигналу до иуму, Q-коефіиієнта, потужності сигналу та потужності шуму). Нарешті, у роботі описано процедури, необхідні для глобальної оптимізації систем МЩПХ. 